\title{
Retrograde en bloc resection for non- muscle invasive bladder tumor can reduce the risk of seeding cancer cells into the peripheral circulation
}

Haichao Huang ${ }^{1+}$, Tao Wang ${ }^{1+}$, Metages Gashaw Ahmed ${ }^{2}$, Lin Zhu ${ }^{2}$, Chaoyong Yang ${ }^{2}$, Wei Li ${ }^{1}$, Zhun Wu ${ }^{1}$, Xuegang Wang ${ }^{1}$, Kaiyan Zhang ${ }^{1 *}$ and Jinchun Xing ${ }^{1 *}$

\begin{abstract}
Objective: To ascertain whether en bloc resection could reduce the risk of seeding cancer cells into the circulation during the resection of non-muscle invasive bladder cancer (NMIBC).

Methods: Patients with primary NMIBC were enrolled in this prospective study from October 2017 to May 2018. Patients were allocated to receive conventional transurethral resection of the bladder (TURB) or retrograde en bloc resection technique of the bladder tumor (RERBT). Blood samples $(1 \mathrm{ml})$ for circulating tumor cell (CTC) enumeration were drawn from the peripheral vein prior to resection (PV1), immediately after resection of the tumor base (PV2), and at $12 \mathrm{~h}$ after resection (PV3). Intra-group comparisons of the changes in the number of CTCs identified among the PV1, PV2, and PV3 blood samples were performed in each group.

Results: A total of 21 patients (12 in the RERBT group and 9 in the TURB group) were recruited. For patients receiving TURB, the level of CTCs identified in PV3 was significantly higher than that in PV1 $(p=0.047)$. However, there was no significant difference in CTC counts before and after resection in the RERBT group.
\end{abstract}

Conclusion: RERBT did not increase the number of tumor cells in the bloodstream.

Keywords: Bladder tumor, Resection, Circulating tumor cells

\section{Micro abstract}

The aim of this study was to ascertain whether en bloc resection could reduce the risk of seeding cancer cells into the circulation during the resection of non-muscle invasive bladder cancer (NMIBC). The present study further verifies the risk of conventional TURB in promoting cell seeding during resection. Moreover, ERBT is an alternative surgical management for endoscopic treatment of NMIBC that may reduce the risk of cell seeding during resection.

*Correspondence: kaiyanzhang_xmu@163.com; xmcua_2007@163.com ${ }^{\dagger}$ Haichao Huang and Tao Wang contributed equally to this work.

'Department of Urology, The First Affiliated Hospital of Xiamen University, 55 Zhenhai Road, Siming District, Xiamen 361003, Fujian, China

Full list of author information is available at the end of the article

\section{Introduction}

Bladder cancer is the second most common type of urological cancer after prostate cancer [1], and transurethral resection of the bladder (TURB) is the standard in the management of non-muscle invasive bladder cancer (NMIBC) [2]. During the process of TURB, a piece-by-piece resection to the muscle layer is performed and the pressure within the bladder exceeds the venous pressure. Therefore, tumor cells may theoretically travel into the venous system during the TURB procedure. Increasing evidence shows that TURB may contribute to the increase in circulating tumor cell (CTC) count in patients with urothelial carcinoma of the bladder (UCB), and en bloc resection of the tumor may be a new approach to address this issue $[3,4]$.

Then, we hypothesized that en bloc resection of the bladder tumor (ERBT) but not piece-by-piece resection performed as TURB would prevent the seeding of tumor 
cells into the circulation during resection. To test this hypothesis, we introduced a novel retrograde en bloc resection of the bladder tumor (RERBT) technique, which has been reported in our previous study [5], and we measured the CTC counts before and after the resection. Moreover, the CTC counts before and after the resection were evaluated in patients who were treated with conventional TURB.

\section{Methods}

\section{Patient and data collection}

Between October 2017 and May 2018, a total of 26 consecutive patients with histologically confirmed primary UCB received transurethral resection (conventional TURB or RERBT, non-randomized) at our institution (The First Affiliated Hospital of Xiamen University). The CTC counts before and after the resection were evaluated. A preoperative flexible cystoscopy with or without biopsy was performed in all patients. To obtain a homogeneous population, the inclusion criteria were as follows: no multifocal tumors (less than 3 tumors) on cystoscopy, tumor size $<3 \mathrm{~cm}$ and $\geq 1 \mathrm{~cm}$ on cystoscopy, and no other malignancy history. Five patients were then excluded from the study, including 2 patients with squamous cell differentiation on postoperative pathological reports, 2 patients with surgery-related bleeding who received re-electrocoagulation within $24 \mathrm{~h}$ after the initial operation, and 1 patient with muscle invasion on the postoperative pathological report (T2 stage).

Of the 21 patients eligible for the final analysis, 9 patients received TURB, while the remaining 12 patients were treated with RERBT. Clinicopathological features, including age, gender, smoking history, tumor size and number, operation duration, and postoperative tumor grade and stage, were collected. The results of histopathology were classified according to the TNM system (the 7th edition) and graded using the WHO 2004 classification. The patients were followed up by cystoscopy every 3 months for up to 13 months of follow-up (5-13 months). Other imaging analyses, including ultrasonography, chest radiography, and abdominal CT, were performed if indicated. Recurrence was defined as intravesical recurrence at any grade and any $\mathrm{T}$ category. This study was reviewed and approved by the Ethics Committee of The First Affiliated Hospital of Xiamen University.

\section{Surgical procedure}

Both RERBT and TURB were performed using the same Circom 25.6F continuous flow resectoscope with a monopolar electrode loop (Richard Wolf GmbH, Knittlingen, Germany) and with cutting and coagulation power set at 110 and $75 \mathrm{~W}$, respectively (Valley Lab, USA). The fluid bag was placed $50 \mathrm{~cm}$ above the bladder intraoperatively and postoperatively. The administration of continuous bladder irrigation was performed for at least $12 \mathrm{~h}$ after the operation for all patients. All operations were performed by 3 senior urologists who had at least 5 years of experience in endoscopic treatment of NMIBC (KYZ, WL, and $\mathrm{ZW}$ ). The final decision on the modality of surgery (TURB vs. RERBT) was reached using a surgeon/patient decision prior to surgery but not a randomization.

The process of the RERBT technique was introduced briefly as follows (Additional file 1: Video S1). Blood vessels entering the tumor were blocked before resection by electro-coagulating the macroscopic normal mucosa approximately 0.5 to $1.0 \mathrm{~cm}$ away from the tumor base to reduce intraoperative hemorrhaging. During the resection, bleeding vessels were coagulated simultaneously, providing a better visualization. Therefore, the time and degree of vascular opening was reduced and the opportunity for tumor cells to enter the bloodstream was kept to a minimum. Most importantly, the tumors were removed en bloc resection in RERBT rather than a piece-by-piece resection as in TURB [5]; there was no tumor chip floating in the bladder during the resection, and continuous bladder irrigation was performed after the resection.

\section{CTC isolation and detection}

Blood samples $(1 \mathrm{ml})$ for CTC enumeration were drawn from the peripheral vein prior to resection (PV1), immediately after resection of the tumor base (PV2), and at $12 \mathrm{~h}$ after resection (PV3). Meanwhile, a total of four volunteers ( 2 males, 2 females) who had no history of malignant tumors were recruited for CTC analysis as a control group. Evacuated EDTA-coated blood collection tubes were used to collect blood samples and then kept at $4{ }^{\circ} \mathrm{C}$ before enumeration. All samples were analyzed within one working day.

We employed a new device, a size-dictated immunocapture chip (SDI-Chip) [6], to measure the CTC counts. Epithelial cell adhesion molecule (EpCAM) and cytokeratin (CK) were used as positive stains, lymphocyte common antigen (CD45) as a negative stain, and 4',6-diamidino-2-phenylindole (DAPI) as a nuclear stain to distinguish tumor cells from leukocytes. With this assay, a CTC was defined as a nucleated cell (DAPI-positive) with CK-positive and CD45negative phenotype (Fig. 1). Enumeration of CTCs was performed by two experienced laboratory researchers (LZ and AMG) who were blinded to the clinicopathological data. In the event of any discrepancies, the researchers re-evaluated the images together until a consensus was reached.

\section{Statistical analysis}

For statistical analysis, numerical variables were compared with $t$ tests and categorical variables were compared with the chi-square test (Fisher exact test) using Statistic Package for the Social Sciences (SPSS) software, version 22.0. In addition, intra-group comparisons of the 


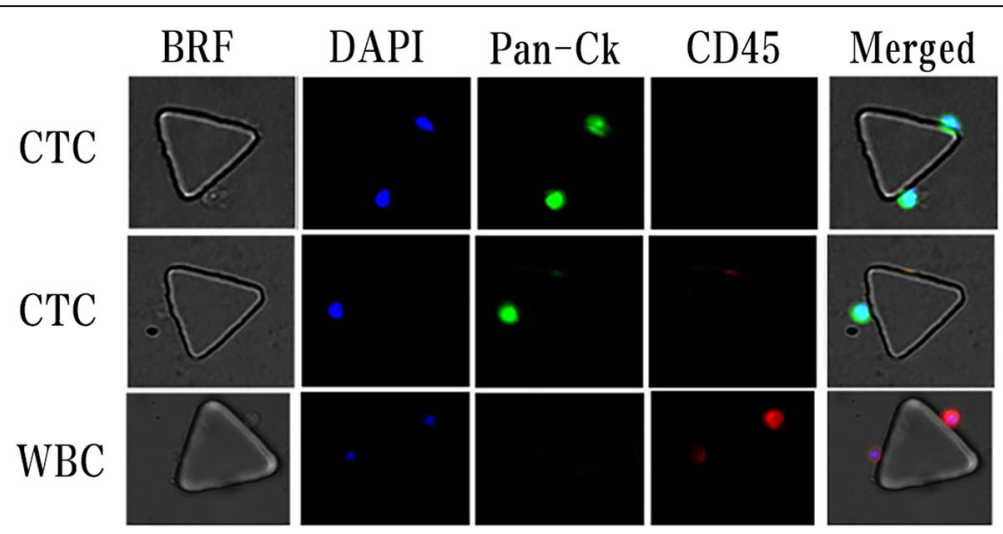

Fig. 1 A CTC is defined as a nucleated cell (DAPI-positive) with CK-positive and CD45-negative phenotype, and a white blood cell is defined as CD45-negative

changes in CTC counts among the PV1, PV2, and PV3 time points were performed by paired $t$ tests. In all tests, a two-tailed $p$ value of $<0.05$ was considered to indicate significance.

\section{Results}

Baseline characteristics and survival outcomes

A total of 21 patients with primary NMIBC were involved in our study; 9 patients were treated with TURB, while the remaining 12 patients received RERBT. There were no statistically significant differences in terms of demographics, smoking history, operation duration, postoperative tumor grade or stage (Table 1), and baseline CTC counts (Table 2) between the two groups. Until November 2018, 2 patients experienced disease recurrence, with a median follow-up period of 8 months (5-13 months). Both patients who experienced disease recurrence were in the TURB group. Survival data

Table 1 Descriptive clinicopathological characteristics of non-muscle invasive bladder cancer patients treated with TURB and RERBT

\begin{tabular}{|c|c|c|c|c|}
\hline Characteristics & $\begin{array}{l}\text { TURB, } n(\%) \\
N=9\end{array}$ & $\begin{array}{l}\text { RERBT, } n(\%) \\
N=12\end{array}$ & Total & $\overline{p \text { value }}$ \\
\hline Age (year) & & & & 0.670 \\
\hline$<60$ & $4(44.4)$ & $7(58.3)$ & 11 & \\
\hline$\geq 60$ & $5(55.6)$ & $5(41.7)$ & 10 & \\
\hline Gender & & & & 1.000 \\
\hline Male & $7(77.8)$ & $9(75.0)$ & 16 & \\
\hline Female & $2(22.2)$ & $3(25.0)$ & 5 & \\
\hline Smoking history & & & & 0.660 \\
\hline No & $3(33.3)$ & $6(50.0)$ & 9 & \\
\hline Yes & $6(66.7)$ & $6(50.0)$ & 12 & \\
\hline Pathologic stage & & & & 0.660 \\
\hline pTa & $6(66.7)$ & $6(50.0)$ & 12 & \\
\hline pT1 & $3(33.3)$ & $6(50.0)$ & 9 & \\
\hline Size (mean, cm) & $1.59 \pm 0.68$ & $1.88 \pm 0.59$ & 21 & 0.301 \\
\hline Grade (WHO 2014) & & & & 1.000 \\
\hline LG & $7(77.8)$ & $10(83.3)$ & 17 & \\
\hline$H G$ & $2(22.2)$ & $2(16.7)$ & 4 & \\
\hline Operation duration (min) & & & & 1.000 \\
\hline$<60$ & $6(66.7)$ & $7(58.3)$ & 13 & \\
\hline$\geq 60$ & $3(33.3)$ & $5(41.7)$ & 8 & \\
\hline Operation duration (mean, min) & $45.44 \pm 15.14$ & $47.83 \pm 14.90$ & 21 & 0.722 \\
\hline Total & $9(42.9)$ & $12(57.1)$ & 21 & \\
\hline
\end{tabular}

TURB transurethral resection of the bladder, RERBT retrograde en bloc TURB, LG low grade, HG high grade 
Table 2 Correlation between preoperative CTC counts and clinicopathological factors

\begin{tabular}{lllll}
\hline Characteristics & $n(\%)$ & $C T C(\bar{x} \pm s / m l)$ & $t$ & $p$ value \\
\hline Age (year) & & & -0.213 & 0.833 \\
$<60$ & $11(52.4)$ & $5.64 \pm 7.31$ & & \\
$\geq 60$ & $10(47.6)$ & $6.40 \pm 9.08$ & & \\
Gender & & & -2.687 & 0.019 \\
Male & $16(76.2)$ & $3.57 \pm 4.97$ & & \\
Female & $5(33.8)$ & $0.00 \pm 0.00$ & & \\
Smoking history & & & -1.735 & 0.099 \\
No & $9(42.9)$ & $2.67 \pm 5.66$ & & \\
Yes & $12(51.1)$ & $8.50 \pm 8.79$ & & \\
Pathologic stage & & & 0.764 & 0.454 \\
PTa & $12(51.1)$ & $7.17 \pm 7.31$ & & \\
PT1 & $9(42.9)$ & $4.44 \pm 9.04$ & & \\
Grade (WHO 2014) & & & -2.497 & 0.022 \\
LG & $17(81.0)$ & $4.12 \pm 6.42$ & & \\
HG & $4(19.0)$ & $14.00 \pm 10.07$ & & \\
Resection method & & & -1.350 & 0.193 \\
TURB & $9(42.9)$ & $3.33 \pm 5.83$ & & \\
RERBT & $12(51.1)$ & $8.00 \pm 9.03$ & & \\
\hline
\end{tabular}

LG low grade, HG high grade, CTC circulating cancer cell, TURB transurethral resection of the bladder, RERBT retrograde en bloc TURB displayed in Kaplan-Meier curves demonstrated no statistically significant differences with respect to recurrencefree survival (RFS) between the TURB and RERBT groups $(p=0.084$, Fig. 2).

\section{Correlation between preoperative CTC counts and clinicopathological factors}

There were 16 males and 5 females in this study, and the male gender was significantly associated with higher preoperative CTC counts compared with the association with the female gender $(p=0.019)$. A similar association was observed between tumor grade and preoperative CTC counts $(n=4.12 \pm 6.42 / \mathrm{ml}$ for patients with lowgrade NMIBC vs. $n=14.00 \pm 10.07 / \mathrm{ml}$ for patients with high-grade NMIBC, $p=0.022$ ). Preoperative CTC counts were not associated with age, smoking history, or tumor stage ( $p=0.833,0.099$, and 0.454 , respectively) (Table 2 ).

\section{Changes in CTCs before and after tumor resection}

To further verify whether TURB may lead to an increase in CTCs in the bloodstream, blood samples were drawn from the peripheral vein before resection (PV1), after resection of the tumor base (PV2), and at $12 \mathrm{~h}$ after resection (PV3). The mean CTCs at PV1, PV2, and PV3 in the TURB group were $3.33 \pm 5.83 / \mathrm{ml}, 11.78 \pm 11.85 / \mathrm{ml}$, and $22.67 \pm 24.25 / \mathrm{ml}$, respectively. Compared with PV1, a significantly higher number of CTCs was observed at PV3 $(p=0.047)$. Although the PV2 sample did not show a significant increase in CTCs compared to PV1, and

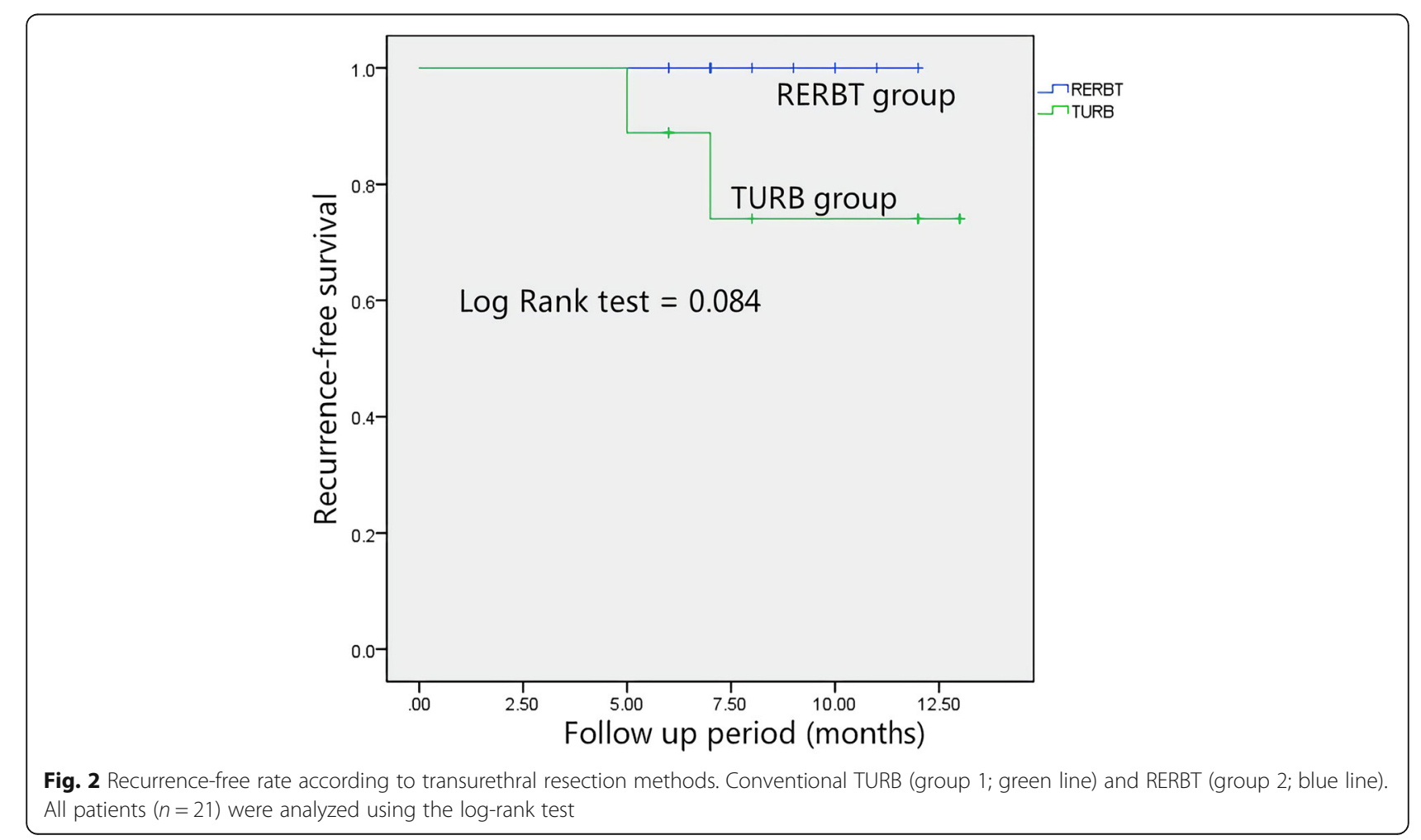


PV3 did not show a significant increase in CTCs compared to PV2 ( $p=0.056$ and 0.068 , respectively), there was an insignificant increasing trend of CTC number during the entire TURB process.

On the other hand, the mean CTCs of PV1, PV2, and PV3 in the RERBT group were $8.00 \pm 9.03 / \mathrm{ml}, 8.00 \pm$ $9.80 / \mathrm{ml}$, and $4.33 \pm 5.52 / \mathrm{ml}$, respectively. There were no significant changes in CTCs among the PV1, PV2, and PV3 samples in the RERBT group (the $p$ values of PV3 vs. PV1, PV2 vs. PV1, and PV3 vs. PV2 were 0.176, 1.000 , and 0.094 , respectively). In addition, for the four control volunteers, no measurable CTCs were observed.

\section{Discussion}

NMIBCs are known as malignant urothelial tumors that do not invade the detrusor and are staged as $\mathrm{Ta}, \mathrm{T} 1$, or carcinoma in situ (CIS). TURB remains the gold standard for the management of NMIBC. However, the pieceby-piece resection pattern violates the basic oncological surgical principles of en bloc resection and may lead to tumor cell dispersal. Despite complete resection, these tumors have a high propensity to recur and progress, with unacceptable rates of recurrence and progression as high as 78 and $45 \%$ within 5 years after the initial TURB, respectively [7]. Does the TURB itself contribute to the poor oncological outcomes for NMIBC, which is traditionally categorized as superficial bladder cancer?

In a recent preliminary study including 17 UCB patients, Engilbertsson and colleagues reported that TURB could cause the seeding of tumor cells into the bloodstream during the process of TURB [3]. This finding has since been validated by another prospective study. In this study, Blaschke et al. showed that the number of CTCs was increased postoperatively in 3 of 8 patients who received TURB for UCB [4]. In our study, 9 patients received conventional TURB for NMIBC, and the CTC counts $12 \mathrm{~h}$ after the resection were significantly higher than the CTC counts before the resection in the bloodstream $(p=0.047)$, which was consistent with the results of the two studies mentioned above $[3,4]$. The underlying mechanisms may lie in the fact that (1) tumors are resected separately in fractions but not resected via en bloc during traditional TURB; (2) the integrity of the bladder wall is damaged; and (3) the pressure within the bladder exceeds the venous pressure during the process of TURB. Taken together, conventional TURB violates the basic oncological surgical tenet of en bloc resection, promoting the spread of the tumor debris and cells into the systemic circulation through damaged vascular walls during the piece-by-piece resection. Further development of novel TURB techniques is needed to overcome these disadvantages. ERBT represents an alternative technique for endoscopic treatment of NMIBC. For tumors of more than $1 \mathrm{~cm}$ in size, ERBT could remove the whole tumor in a single piece [8], and it is available to retrieve whole tumor specimens up to $4.5 \mathrm{~cm}$ in size [9]. ERBT has the potential to overcome tumor fragmentation and possible consequences including cell seeding and low-qualified specimens, which are the biggest limitations of conventional TURB.

To test whether ERBT could reduce the risk of cell seeding, we introduced a novel RERBT technique [5] and compared the number of CTCs identified before and after resection using a size-dictated immunocapture chip, which had been reported in our previous study [6]. In the current study, all the tumors were less than $3 \mathrm{~cm}$ in size, so we were able to retrieve the tumor in one piece when ERBT was performed. As a result, there was no tumor debris during the resection process. After ERBT, tumor cells may have less chance to remain in the bladder and further insert into the circulation during irrigation. In contrast, when conventional TURB was performed, tumor cells are more inclined to remain in the bladder after resection, leading to a further increase in the number of CTCs. Moreover, timely electrocoagulation of the bleeding vessels was performed due to better visualization during the ERBT, which may contribute to a decrease in the risk of cell seeding through damaged vascular walls. Thus, there was no significant difference in the changes in CTCs identified among the PV1, PV2, and PV3 time points in patients who were treated with RERBT, which was in contrast with the results of patients treated with conventional TURB, where a significant increase in the CTC counts was observed.

Although whether ERBT, compared with conventional TURB, could provide a better long-term survival for patients with NMIBC is controversial, a recent metaanalysis in which patients who received ERBT showed a significantly lower 24-month recurrence rate than that of patients who received conventional TURB [10]. In our study, 2 patients who experienced disease recurrence were in the TURB group. During the follow-up period, no patients in the RERBT group experienced disease recurrence. However, there were no significant differences with respect to RFS between the TURB and RERBT groups $(p=0.084)$, which may be due to the relatively shorter survival period. Incomplete resection, cell implantation, or tumor biology itself may contribute to these results in terms of recurrence rate. Moreover, in a recent, small prospective study, the progression rate, though without significance, was lower in patients who received ERBT than in patients who received conventional TURB [11]. We suggested that the improvement in terms of the progression rate may be due to the reduction in cell seeding during the process of resection when ERBT was performed. Based on a series of studies, the presence of CTCs has been confirmed to be associated with a variety of solid malignancies, including breast, 
colorectal, gastric, and prostate cancer [12-15]. Although inconsistent results with regard to the association between the clinicopathological features and the presence of CTCs in patients with UCB have been reported [16-21], the association between the detection of CTCs and poor oncological outcomes of UCB patients has already been confirmed by a variety of studies [18-20,22]. Recently, a systemic review study regarding the association between CTC-positive results and the clinicopathological features and prognostic outcomes in 2161 patients with UCB showed that the presence of CTCs in the peripheral blood was an independent predictive indicator of both poor histopathological parameters and poor oncological outcomes [23]. Furthermore, in the first report regarding the presence of CTCs in NMIBC patients using CellSearch, Gazzaniga et al. showed that the detection of CTCs was significantly associated with both poor histopathological parameters and poor oncological outcomes [18]. Similar results were also observed in a recent study by Gradilone and colleagues. In this study, which included 54 histologically confirmed T1G3 UCB patients, the detection of CTCs in the peripheral blood circulation was an independent risk factor for poor disease-free survival [22]. Similarly, in our study, patients with histopathologically confirmed high-grade NMIBC had a significantly higher level of preoperative CTCs than did patients with low-grade NMIBC $(p=0.022)$. Therefore, CTCs play an important role in the survival of UCB patients. However, whether the difference in the changes in CTCs during tumor resection contributes to a higher progression rate in patients who received conventional TURB than in patients who received ERBT was unclear, as it is an extremely complex process from cell seeding to micrometastasis and distant metastasis. Further studies are needed to validate this hypothesis in the future.

There are two main limitations of this study. First, it is not a randomized study. However, we enrolled a homogeneous population, and there was no significant difference between RERBT and conventional TURB groups in the distribution of clinicopathological features, including tumor grade which was associated with the presence of CTCs. Moreover, there was no significant difference in the preoperative CTC counts between the TURB group and the RERBT group $(p=0.193)$. The difference in changes in CTC counts before and after resection was evaluated using an intra-group comparison. The results of one group would not affect the results of the other group. Second, the limited sample size was also a limitation of our preliminary study. As the cost of CTC enumeration once was almost $\$ 1000$ in China, so at least $\$ 3000$ was required for one patient in our study. CTCs are unlike other simple and inexpensive biomarkers that can be evaluated on a large scale without considering the cost-effect in a preliminary study. We believe that this cost-effect issue has been taken into consideration in studies from western populations [3, 4]. There are still several limitations of our study. First, all resections were performed by three surgeons. Second, we enrolled patients without multifocal NMIBC in the present study. In addition, the tumors enrolled were sized as $<3 \mathrm{~cm}$ and $\geq 1 \mathrm{~cm}$ on cystoscopy. Thus, whether RERBT remains a prophylactic effect for multifocal tumors or tumors of a larger size is unclear. However, to the best of our knowledge, we are the first to demonstrate that RERBT did not increase the number of cells in the bloodstream compared to conventional TURB, which may be a potential reason to explain the better oncological outcomes of patients who received ERBT than the outcomes of patients who were treated with conventional TURB $[10,11]$. We believe our preliminary study will encourage other groups conducting further prospective studies with larger sample sizes to gain insight into this field. Moreover, we hope our preliminary study will support other groups who are committed to improving the transurethral resection techniques and upholding oncological principles in the endoscopic management of bladder tumors.

\section{Conclusion}

The present study further verifies the risk of conventional TURB in promoting cell seeding during resection. Moreover, ERBT is an alternative surgical management for endoscopic treatment of NMIBC that may reduce the risk of cell seeding during resection. To our knowledge, these results are novel.

\section{Supplementary information}

Supplementary information accompanies this paper at https://doi.org/10. 1186/s12957-020-1808-0.

Additional file 1: Video S1. A patient with a 1.6-cm-diameter lesion underwent RERBT.

\section{Acknowledgements}

We thank Prof. Hua Xu of the Department of Urology, Tongji Hospital, Tongji Medical School, Huazhong University of Science and Technology, for providing editorial and professional writing assistance in the preparation of this manuscript.

\section{Authors' contributions}

Protocol development: HH, JX and KZ; Data collection: TW, MGA, LZ, CY, WL, ZW and XW; Data analysis: $\mathrm{HH}$, TW and $\mathrm{KZ}$; Manuscript writing: $\mathrm{HH}$; Critical revision: JX, CY and $\mathrm{KZ}$. All authors read and approved the final manuscript.

\section{Funding}

This work was supported by the Fujian Provincial Department of Science and Technology (Project Number: 2016D009; Recipient: Jinchun Xing) and by Xiamen Science and Technology Plan (Project Number: 3502Z20184014; Recipient: Jinchun Xing)

Availability of data and materials Research data are not shared. 


\section{Ethics approval and consent to participate}

This study was reviewed and approved by the Ethics Committee of The First Affiliated Hospital of Xiamen University.

\section{Consent for publication}

Informed consents were obtained from all the patients.

\section{Competing interests}

The authors declare that they have no competing interests.

\section{Author details}

'Department of Urology, The First Affiliated Hospital of Xiamen University, 55 Zhenhai Road, Siming District, Xiamen 361003, Fujian, China. ${ }^{2}$ MOE Key Laboratory of Spectrochemical Analysis and Instrumentation, Key Laboratory of Chemical Biology of Fujian Province, State Key Laboratory of Physical Chemistry of Solid Surfaces, Collaborative Innovation Center of Chemistry for Energy Materials, Department of Chemical and Biochemical Engineering, Department of Chemical Biology, College of Chemistry and Chemical Engineering, Xiamen University, Xiamen 361003, Fujian, China.

Received: 22 October 2019 Accepted: 27 January 2020

Published online: 10 February 2020

\section{References}

1. Siegel R, Miller K, Jemal A. Cancer statistics, 2017. CA Cancer J Clin. 2017:67:7-30

2. Gontero P, Sylvester R, Pisano F, et al. Prognostic factors and risk groups in T1G3 non-muscle-invasive bladder cancer patients initially treated with Bacillus Calmette-Guerin: results of a retrospective multicenter study of 2451 patients. Eur Urol. 2015;67:74-82.

3. Engilbertsson $\mathrm{H}$, Aaltonen KE, Björnsson S, et al. Transurethral bladder tumor resection can cause seeding of cancer cells into the bloodstream. J Urol. 2015;193:53-7.

4. Blaschke S, Koenig F, Schostak M. Hematogenous tumor cell spread following standard transurethral resection of bladder carcinoma. Eur Urol. 2016;70:544-5

5. Zhang KY, Xing JC, Li W, et al. A novel transurethral resection technique for superficial bladder tumor: retrograde en bloc resection. World J Surg Oncol. 2017:15:125.

6. Ahmed MG, Abate MF, et al. Isolation, detection, and antigen-based profiling of circulating tumor cells using a size-dictated immunocapture chip. Angew Chem Int Ed Engl. 2017:56:10681-5.

7. van den Bosch S, Witjes JA. Long-term cancer-specific survival in patients with high-risk, non-muscle-invasive bladder cancer and tumour progression: a systematic review. Eur Urol. 2011;60:493-500.

8. Kramer MW, Wolters M, Herrmann TR. En bloc resection of bladder tumors: ready for prime time? Eur Urol. 2016;69:967-8.

9. Naselli A, Introini C, Germinale F, et al. En bloc transurethral resection of bladder lesions: a trick to retrieve specimens up to $4.5 \mathrm{~cm}$. BJU Int 2012;109:960-3.

10. Wu YP, Lin TT, Chen SH, et al. Comparison of the efficacy and feasibility of en bloc transurethral resection of bladder tumor versus conventional transurethral resection of bladder tumor: a meta-analysis. Medicine. 2016;95:e5372.

11. Sureka SK, Agarwal V, Agnihotri S, et al. Is en-bloc transurethral resection of bladder tumor for non-muscle invasive bladder carcinoma better. Indian J Urol. 2014:30:144-9.

12. Huang X, Gao P, Sun J, et al. Clinicopathological and prognostic significance of circulating tumor cells in patients with gastric cancer: a meta-analysis. Int J Cancer. 2015;136:21-33.

13. Lv Q, Gong L, Zhang T, et al. Prognostic value of circulating tumor cells in metastatic breast cancer: a systemic review and meta-analysis. Clin Transl Oncol. 2016;18:322-30

14. Rahbari NN, Aigner M, Thorlund K, et al. Meta-analysis shows that detection of circulating tumor cells indicates poor prognosis in patients with colorectal cancer. Gastroenterology. 2010;138:1714-26.

15. de Bono JS, Scher HI, Montgomery RB, et al. Circulating tumor cells predict survival benefit from treatment in metastatic castration-resistant prostate cancer. Clin Cancer Res. 2008;14:6302-9.
16. Flaig TW, Wilson S, van Bokhoven A, et al. Detection of circulating tumor cells in metastatic and clinically localized urothelial carcinoma. Urology. 2011;78:863-7.

17. Naoe M, Ogawa Y, Morita J, et al. Detection of circulating urothelial cancer cells in the blood using the CellSearch system. Cancer. 2007;109:1439-45.

18. Gazzaniga P, Gradilone A, de Berardinis E, et al. Prognostic value of circulating tumor cells in nonmuscle invasive bladder cancer: a CellSearch analysis. Ann Oncol. 2012;23:2352-6.

19. Abrahamsson J, Aaltonen $\mathrm{K}$, Engilbertsson $\mathrm{H}$, et al. Circulating tumor cells in patients with advanced urothelial carcinoma of the bladder: association with tumor stage, lymph node metastases, FDG-PET findings, and survival. Urol Oncol. 2017;35:606.e9-16.

20. Rink M, Chun FK, Dahlem R, et al. Prognostic role and HER2 expression of circulating tumor cells in peripheral blood of patients prior to radical cystectomy: a prospective study. Eur Urol. 2012;61:810-7.

21. Guzzo TJ, McNeil BK, Bivalacqua TJ, et al. The presence of circulating tumor cells does not predict extravesical disease in bladder cancer patients prior to radical cystectomy. Urol Oncol. 2012;30:44-8.

22. Gradilone A, Petracca A, Nicolazzo C, et al. Prognostic significance of survivin-expressing circulating tumour cells in T1G3 bladder cancer. BJU Int. 2010;106:710-5.

23. Zhang Z, Fan W, Deng $Q$, et al. The prognostic and diagnostic value of circulating tumor cells in bladder cancer and upper tract urothelial carcinoma: a meta-analysis of 30 published studies. Oncotarget. 2017;8: 59527-38.

\section{Publisher's Note}

Springer Nature remains neutral with regard to jurisdictional claims in published maps and institutional affiliations.

Ready to submit your research? Choose BMC and benefit from:

- fast, convenient online submission

- thorough peer review by experienced researchers in your field

- rapid publication on acceptance

- support for research data, including large and complex data types

- gold Open Access which fosters wider collaboration and increased citations

- maximum visibility for your research: over $100 \mathrm{M}$ website views per year

At BMC, research is always in progress.

Learn more biomedcentral.com/submissions 